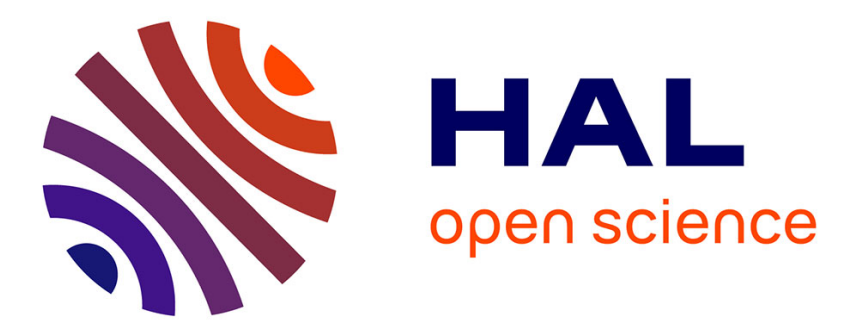

\title{
Elucidating the effect of bimodal grain size distribution on plasticity and fracture behavior of polycrystalline materials
}

Fabrice Barbe, Ivano Benedetti, Vincenzo Gulizzi, Mathieu Calvat, Clément Keller

\section{To cite this version:}

Fabrice Barbe, Ivano Benedetti, Vincenzo Gulizzi, Mathieu Calvat, Clément Keller. Elucidating the effect of bimodal grain size distribution on plasticity and fracture behavior of polycrystalline materials. Journal of Multiscale Modelling, In press, 10.1142/S1756973720500079 . hal-02922183

\section{HAL Id: hal-02922183 \\ https://hal.science/hal-02922183}

Submitted on 1 Sep 2020

HAL is a multi-disciplinary open access archive for the deposit and dissemination of scientific research documents, whether they are published or not. The documents may come from teaching and research institutions in France or abroad, or from public or private research centers.
L'archive ouverte pluridisciplinaire HAL, est destinée au dépôt et à la diffusion de documents scientifiques de niveau recherche, publiés ou non, émanant des établissements d'enseignement et de recherche français ou étrangers, des laboratoires publics ou privés. 


\title{
Elucidating the effect of bimodal grain size distribution on plasticity and fracture behavior of polycrystalline materials
}

\author{
Fabrice Barbe, 1,a) Ivano Benedetti, 2,b) Vincenzo Gulizzi, 3, (c) Mathieu Calvat, 1, d) \\ and Clément Keller ${ }^{1, e)}$ \\ 1) INSA Rouen, UNIROUEN, Groupe de Physique des Matériaux, Normandie Univ, UMR CNRS 6634, Rouen, F-76000, France \\ ${ }^{2)}$ Department of Engineering, University of Palermo, Viale delle Scienze, Edificio 8, 90128, Palermo, Italy \\ ${ }^{3)}$ Center for Computational Sciences and Engineering (CCSE), Lawrence Berkeley National Laboratory, MS 50A-3111, Berkeley, \\ CA 94720, USA

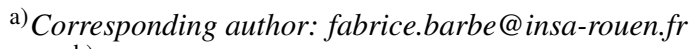

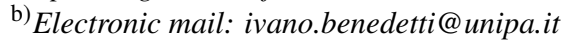 \\ ${ }^{c}$ Electronic mail: vgulizzi@lbl.gov \\ ${ }^{\mathrm{d})}$ Electronic mail: mathieu.calvat@insa-rouen.fr \\ e) Electronic mail: clement.keller@insa-rouen.fr
}

\begin{abstract}
The refinement of grains in a polycrystalline material leads to an increase in strength but as a counterpart to a decrease in elongation to fracture. Different routes are proposed in the literature to try to overpass this strength-ductility dilemna, based on the combination of grains with highly contrasted sizes. In the simplest concept, coarse grains are used to provide relaxation locations for the highly stressed fine grains. In this work, a model bimodal polycrystalline system with a single coarse grain embedded in a matrix of fine grains is considered. Numerical full-field micro-mechanical analyses are performed to characterize the impact of this coarse grain on the stress-strain constitutive behaviour of the polycrystal: the effect on plasticity is assessed by means of crystal plasticity finite element modelling [1] while the effect on intergranular fracture behaviour is studied by using boundary element modelling [2]. The analysis of the computational results, compared to the experimentally characterised tensile properties of a bimodal 316L stainless steel, suggests that the elasto-plastic interactions taking place prior to micro-cracking may play an important role in the mechanics of fracture of this steel.
\end{abstract}

\section{INTRODUCTION}

As characterized in the seminal works of Hall and Petch [3, 4], the yield stress and strength of a metal alloy is generally inversely proportional to the square root of the mean Grain Size (GS). This observation can be made for most metal alloys and for grain sizes larger than $1 \mu \mathrm{m}$. Reducing the grain size however also results in the degradation of the ductility as could be characterised in [1, 5, 6]. This has motivated the development of different strategies to overpass this strength-ductility dilemna by introducing in the material some microstructural characteristics acting as local stress relaxers. Tailoring the grain size distribution is one of these strategies [7, 8, 9] and one remarkable case corresponds to the bimodal GS distribution, where Coarse Grains (CG) are present in a matrix of Fine Grains (FG).

One of the most efficient means to elaborate these kind of architected materials is the sintering of metal powders [1, 10, 11, 12, 13]. It indeed enables to control the main metrics of a bimodal microstructure, viz. the mean GS of the CG population, the mean GS of the FG population, and the volume fraction of CG. In the experimental work [1, 14], different associations of these three metrics in a $316 \mathrm{~L}$ austenitic stainless steel have been considered from the spark plasma sintering to the tensile fracture properties. Whereas unimodal cases confirmed the reduction of the elongation at break as GS was decreased, bimodal cases have shown that a good combination of strength and ductility could be obtained for a GS ratio larger than 10 and a FG size of the order of few $\mu m$.

Different modelling approaches have been proposed in the literature to predict the mechanical properties according to the GS distribution and to study the interactions taking place between FG and CG. Some of these proposals rely on mean field hypotheses of self-consistent homogenization approaches [15, 16, 17] or on the idealization of a GS population as a homogeneous phase [18], or are restricted to the early stages of plastic deformation [19]. In the study [20], a comprehensive presentation of a full field crystal plasticity modelling applied to bimodal polycrystals has been provided. Yet, although this modelling approach provides a very fine description of the elastoplastic mechanisms originally taking place in a bimodal polycrystal, its applicability is restricted to crystal plasticity. The present study aims at constructing the bases of a full field modelling approach accounting for both crystal plasticity and microcracking. For such preliminary steps, the micro-mechanics of a model bimodal polycrystal is studied considering first its crystal plasticity, by the finite element method, and then its intergranular fracture behaviour, by means of a boundary element framework [21, 22]. 

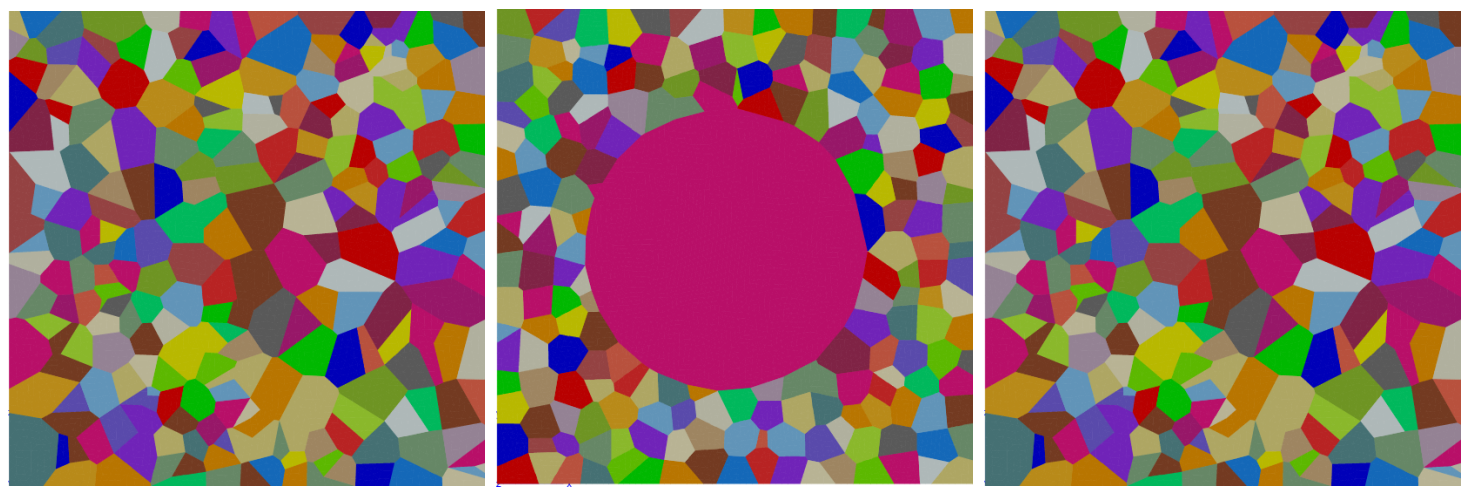

FIGURE 1: The three considered polycrystalline model microstructures. (From left to right) Unimodal Coarse Grain (grain size $5 \mu \mathrm{m})$; Bimodal, with a single CG $(5 \mu \mathrm{m})$ embedded in a matrix of FG $(0.5 \mu \mathrm{m})$; Unimodal Fine Grain (grain size $0.5 \mu \mathrm{m}$ ).

\section{MODELLING}

\section{Material and Microstructure}

The material under concern is a 316L stainless steel elaborated by spark plasma sintering. Its microstructure and tensile properties have been presented in [1] for different combinations of grain sizes. As illustrated by EBSD scans presented therein, CG are commonly observed to be embedded in the matrix of FG as isolated inclusions. Previous numerical analyses [20] have shown that in such a configuration of isolated CG, a domain containing at least 9 CG should be considered to obtain quantitatively representative macroscopic properties; yet a 2D domain containing a single CG could provide qualitativaly representative properties, especially for the analyses of local CG-FG interactions. This is the retained configuration of study in this paper, also motivated by the reduction in computational resources and the comfort of analysis it provides.

Three model polycrystals are considered (fig. 1): a bimodal polycrystal with GS of $5 \mu \mathrm{m}$ and $0.5 \mu \mathrm{m}$ resp. for CG and FG families and a unimodal microstructure containing 256 grains and with a mean GS corresponding to either CG or FG. They result from Laguerre-Voronoi tessellations with specific settings on the nucleation and growth processes [20, 23]. The Neper software was used to generate these microstructure and to obtain the finite elements meshes [24, 25].

\section{Crystal plasticity}

The Schmid-based crystal plasticity constitutive model of Méric and Cailletaud is used [26]. The plastic slip rate $\dot{\gamma}^{s}$ of the slip system $s$ is deduced from the resolved shear stress $\tau^{s}$ and the isotropic hardening $r^{s}$ through the Norton power law 1 where $K$ and $n$ control the rate sensitivity:

$$
\dot{\gamma}^{s}=\left(\frac{\left|\tau^{s}\right|-r^{s}}{K}\right)^{n} \operatorname{signe}\left(\tau^{s}\right)
$$

The hardening component $r^{s}$ evolves according to the saturation law 2 expressed in terms of $p$ the cumulated plastic strain:

$$
r^{s}=R_{0}+Q \sum_{i=1}^{N} h^{s i}\left(1-e^{-b p^{i}}\right) \quad \text { with } \quad \dot{p}^{i}=\left|\dot{\gamma}^{i}\right|
$$

The matrix $h^{s i}$ describes the interactions between slip systems, $Q$ and $b$ affect the rate of saturation and $R_{0}$ is the critical resolved shear stress, above which slip is activated. Hall-Petch-type grain size dependence of the macroscopic yield stress is introduced directly on the scale of the slip system with the definition of $R_{0}$ given in eq. 3 . 


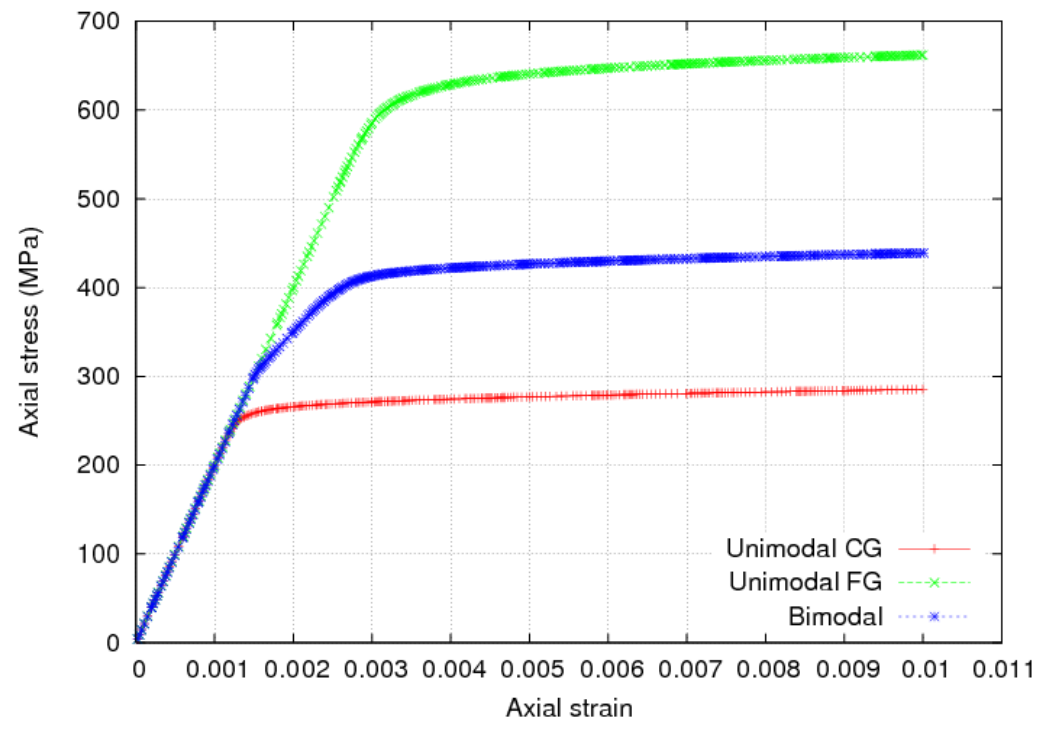

FIGURE 2: Tensile stress-strain curves of the three polycrystals.

$$
R_{0}=r_{0}+\frac{k}{\sqrt{d}}
$$

$d$ is the grain diameter, $k$ controls the dependence to the grain size and $r_{0}$ is the critical resolved shear stress in a grain having large dimensions.

All the material parameters have been identified on the basis of experimental tensile stress-strain curves of the considered 316L steel elaborated by spark plasma sintering, for different mean GS of unimodal polycrystals. It is worth noting that the Hall-Petch dependence introduced in the slip system scale results also in a Hall-Petch dependence of the macroscopic scale of a unimodal polycrystal [20].

\section{Elastoplastic behaviour}

The polycrystals are tensile strained to $1 \%$. The related axial stress and strain responses are presented on two scales: macroscopic behaviour in fig. 2 and field contours in fig. 3 . The presence of the CG in the FG phase, although it occupies only $27 \%$ in volume, significantly decreases the macroscopic stress response. This can be explained from the role that the CG plays in the bimodal system: the CG is not only the base of highest strains localisation, it is responsible for a significant decrease of stress in the FG at its upper and lower neighbourhood: in this relaxation channel, the stresses in the FG population is much lower than in the unimodal FG polycrystal.

The effect of the presence of the CG within the FG matrix is further analysed by means of the mean responses per grain in fig. 4. the case of the bimodal distribution is compared to the case of the unimodal FG distribution. According to the grains stress-strain curves, a large dispersion of the tensile responses is observed in both cases, and FG grains do not seem to reach higher stresses in the bimodal case than in the unimodal case. The statistical distributions in the inserts show that the presence of the CG significantly broadens the dispersion of stresses, creating a bimodal distribution in the range of $60 \%$ to $160 \%$ from the macroscopic bimodal polycrystal stress. It also increases the number of FG grains at both very small strains $(\sim 25 \%$ the mean polycrystal strain) and large strain $(\sim 150 \%$ the mean polycrystal strain).

To quantify the mean effect of the CG on the FG behavior, we consider in fig 5 the mean behavior of each grain population in the bimodal polycrystal (resp. the CG and the FG population) divided by its corresponding mean properties in its counterpart unimodal polycrystal. This is done for each considered variable: axial strain and axial stress. One can then observe the particular behavior of the CG as it interplays with the FG grains: $(i)$ a strain which 


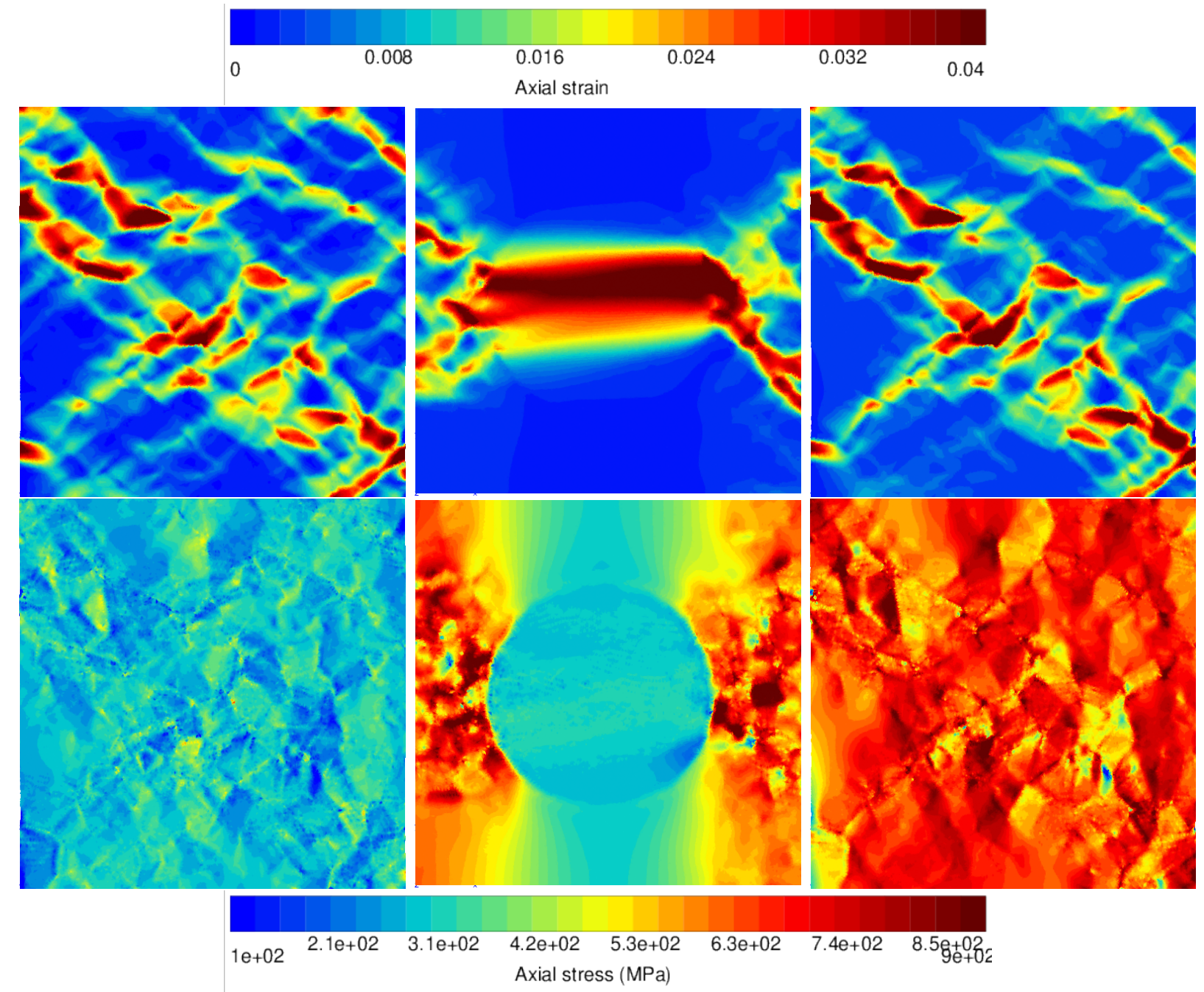

FIGURE 3: Spatial distributions of strain (top) and stress (bottom) at 1\% imposed strain: from left to right, Unimodal CG, Bimodal, Unimodal FG

reaches $180 \%$ the mean strain reached in the unimodal CG polycrystal, but which however remains in a range that many grains reach in the unimodal CG polycrystal (cf. fig. (4), and (ii) a stress which abruptly increases to $120 \%$ and then slowly decreases to $110 \%$ the mean unimodal CG polycrystal. The coincident effect of this CG behavior on the FG population is a decrease to $62 \%$ and $78 \%$ the mean strain and stress (resp.) of the unimodal FG polycrystal.

These increases or decreases of the mean behavior of a grain population in the bimodal system with respect to what it would be in a unimodal configuration results in the intermediate macroscopic stress-strain response given in fig. 2 as could be expected in any kind of "soft inclusion"-"hard matrix" composite. A peculiarity is however observed concerning the early stages of plasticity: three deformation regimes corresponding to (1) elasticity, (2) a first elastoplastic hardening regime and (3) a second elastoplastic hardening regime. These three stages can be distinguished on the curves of stress, strain and plasticity evolutions in each grain population (fig. 5. "plasticity" corresponds to the sum of the slips on the slip systems and thus quantifies a mean plastic slip activity): plasticity in the CG of the bimodal polycrystal starts at higher imposed strain than in the unimodal CG polycrystal and this moment of the load corresponds both to the CG stress relaxation and to the transition from the first to the second deformation regime; the second regime lasts until the FG stress saturates to $78 \%$ the mean stress in the FG unimodal polycrystal. It is further noticed that during the second regime, FG of the bimodal polycrystal display much more plasticity than in the unimodal configuration. It actually starts halfway during the second regime. 

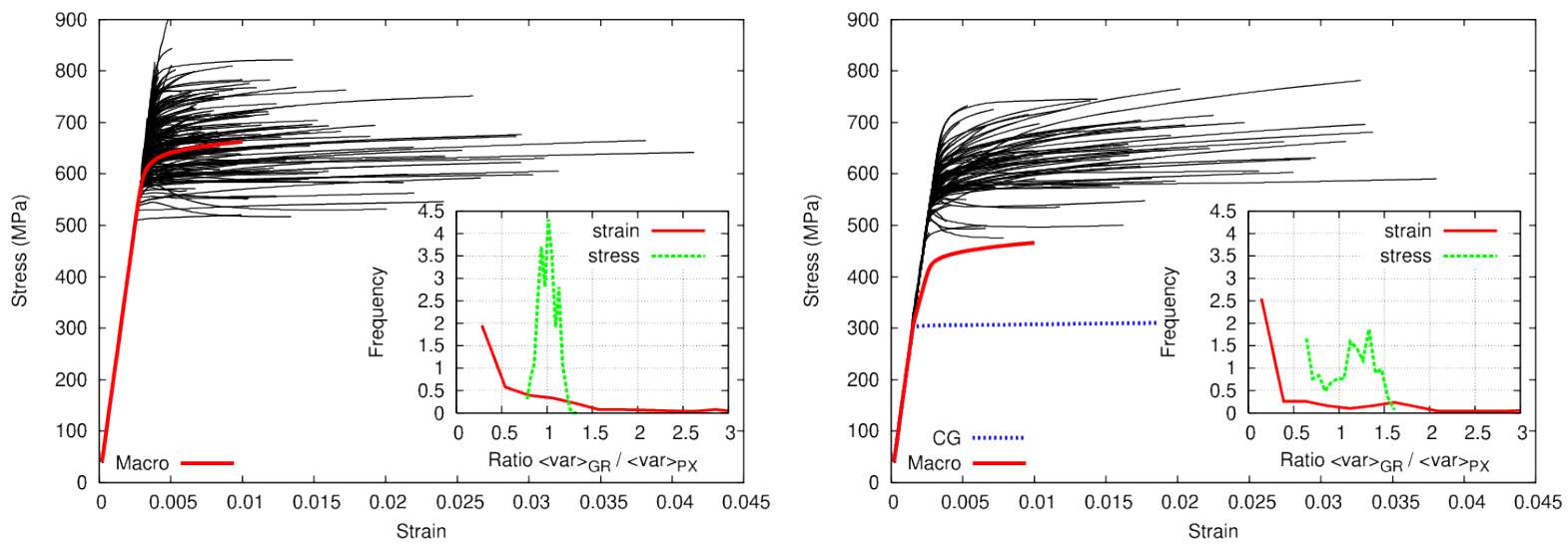

FIGURE 4: Stress-strain curves of all the grains in the Unimodal FG polycrystal (left) and in the Bimodal polycrystal. The inserts present the distribution of the grains stresses and strains at the end of the load. Each grain response (subscript "GR") has been normalized by its corresponding mean polycrystal response (subscript "PX").

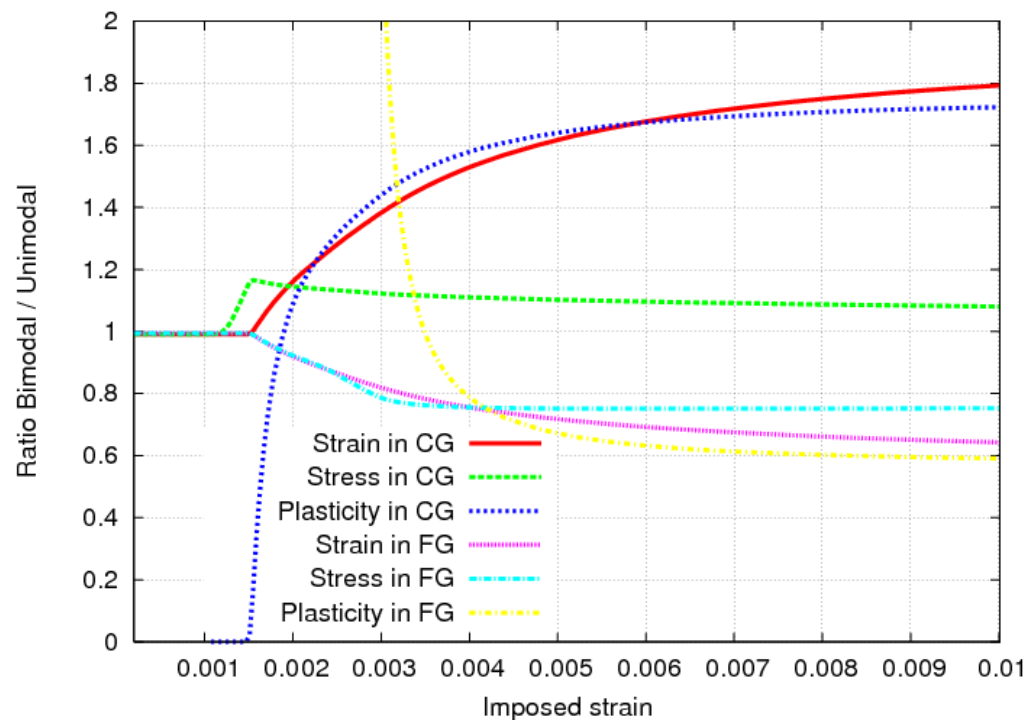

FIGURE 5: The ratio of the mean CG (resp. FG) response in the bimodal polycrystal to the mean response of its unimodal counterpart is plotted against the imposed axial strain. As an example, the mean CG strain in the bimodal polycrystal is the same as in the CG unimodal polycrystal for a strain less than 0.0015 and becomes 1.8 times larger at the end of the test.

\section{Intergranular fracture propagation}

To help elucidate the behaviour of polycrystalline aggregates with a bimodal grain size distribution, the analyses are completed by an investigation into their fracture behaviour, employing a recently developed computational framework based on the use of a boundary integral representation of the polycrystalline micro-mechanics in conjunction with cohesive-frictional interfaces [21, 22]. The method, which is built on a Laguerre-Voronoi representation of the microstructure, allows to study the progressive degradation and micro-cracking of aggregates subjected to quasi-static loading. It employs the boundary element method [27] for discretising the so-called displacements boundary integral equations associated with, or collocated at, the mesh nodes of the discretised individual grains. The process, whose 
details are described in Refs. [22, 28], leads to an aggregate system of equations of the form

$$
\left[\begin{array}{c}
\mathbf{A} \cdot \mathbf{X} \\
\mathbf{I}\left(\mathbf{X}, \boldsymbol{d}^{*}\right)
\end{array}\right]=\left\{\begin{array}{c}
\mathbf{B} \cdot \mathbf{Y}(\lambda) \\
\mathbf{0}
\end{array}\right\}
$$

where the matrix blocks $\mathbf{A}$ and $\mathbf{B}$ collect constant entries stemming from the boundary element numerical integration, the vector $\mathbf{X}$ collects unknown components of displacements and tractions associated with points lying on the surfaces of the individual grains, and it is then suitable for representing intergranular degradation and micro-cracking processes, the vector $\mathbf{Y}$ collects known components of displacements or tractions associated with points lying on the external surface of the aggregate, thus stemming from the boundary conditions, and the non-linear matrix block $\mathbf{I}\left(\mathbf{X}, \boldsymbol{d}^{*}\right)$ implements the intergranular cohesive-frictional laws, accounting also for the loading history through an irreversible damage field whose nodal values associated with the interface pairs are collected within in the vector $\boldsymbol{d}^{*}$. The methodology allows to study the microstructural evolution capturing the transition of individual interfaces from a pristine to a damaged and eventually cracked status. It has been successfully employed to model quasi-static micro-cracking [28, 29, 30], small-strains crystal plasticity [31], hydrogen embrittlement [32], high-cycle fatigue [33], micro-cracking of piezoelectric aggregates [34] and multi-scale modelling of polycrystalline components [35].

In this work, the framework is employed to contribute to better understanding how the bimodal grain size distribution may affect the micro-mechanics of this class of aggregates. At this stage, no crystal plasticity is considered in the boundary element model and the individual grains behave linearly, with the non-linear processes confined at the intergranular interfaces only: this is done to obtain a preliminary assessment of purely morphological effects on the micro-mechanics of cracking, while more general effects will be included extending the study presented in Ref. [31].

Fig. 6 shows the comparison between the micro-cracking patterns obtained for the unimodal FG polycrystal and the bimodal polycrystal. The FG populations have the same mean grain size. The two polycrystals are subjected to a tensile load along the vertical direction, enforced in displacement control conditions. In the unimodal case, if the same cohesive properties are assigned to all the intergranular interfaces, the crack initiates at the grain boundaries lying on planes orthogonal with respect to the loading direction. The interplay between grains anisotropy and orientation mismatch may render some interfaces more susceptible to crack initiation, although the interface orientation remain the dominating initiation factor (in the mentioned modelling assumptions). In the bimodal case, the micro-cracking starts at the interfaces between the larger grain and the smaller ones lying at the top or bottom of the large grain: this is consistent with the unimodal analyses as, in the considered morphology, such interfaces lye on planes normal to the loading direction. After initiation, the micro-cracks follow the border of the large inclusion up to locations at which the external loads starts aligning with the interface, thus reducing the effective traction on the interface. In the bimodal case, also multiple cracking is observed.

Fig. 7 shows the macro stress-strain curves obtained from the two analysed cells. In the adopted assumptions, a light effect of the bimodal morphology is detectable only on the softening branch of the curves. It is apparent that the absence of plastic effects has an important effect on such curves, as all the dissipative processes (damage) are confined over the intergranular interfaces, instead than within all the volume (plasticity). More comprehensive investigations will include the effect of crystal plasticity in the boundary element framework.

\section{CONCLUSION}

The mechanical problem of a single Coarse Grain (CG) embedded in a matrix of Fine Grains (FG) has been considered. It is inspired from the experimental realisations of $316 \mathrm{~L}$ stainless steel polycrystals with bimodal grain size distribution, which have led to an enhancement of the combination of strength and deformability by comparison to unimodal polycrystals. In this set configuration of study, crystal plasticity multiscale analyses show that interactions between the CG and the FG could not be returned to a simple mixture rule of the properties: with the presence of the CG, a large stress relaxation channel appears along the tensile direction, high strains localize within the CG, stress distribution is broadened, plasticity activity starts at lower imposed strain and elastoplastic hardening evolves according to two distinct regimes.

This new situation exercised by the FG can have different consequences on the fracture properties, depending on the mechanisms controlling damage and fracture. The investigations on the intergranular fracture are based on the assumptions of an elastic linear behaviour assigned to the grains and a dissipative process related to damage at grain boundaries. They show that the presence of the CG does not significantly modify the macroscopic inception of softening as compared to the unimodal case. This can be explained by the fact that, in the adopted framework, the orientation of a grain boundary with respect to the tensile direction is a dominant factor in the micro-cracking process, 


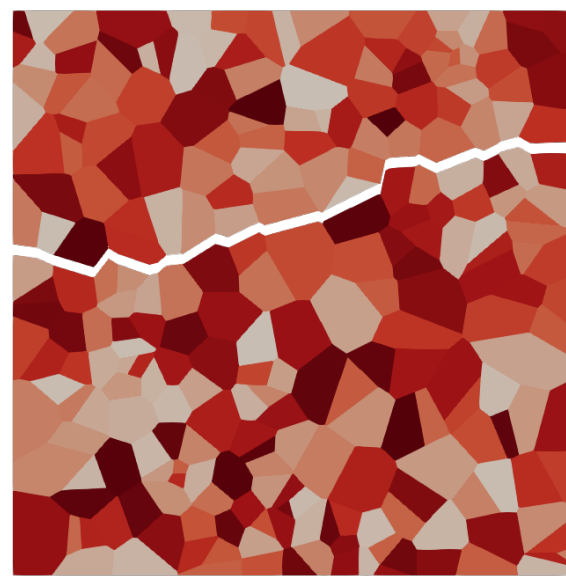

(a)

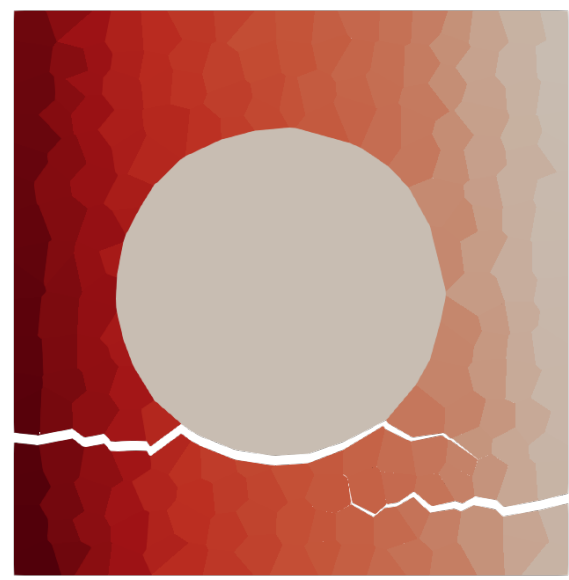

(b)

FIGURE 6: Micro-cracking patterns computed with the boundary element framework for polycrystalline micro-mechanics in Refs. [22, 28] for $(a)$ the unimodal and $(b)$ the bimodal polycrystal. The present simulations do not consider crystal plasticity effects and provide a preliminary assessment of morphological effects of the bimodal distribution on the micro-cracking mechanics in case of linear elastic grains with damaging interfaces.

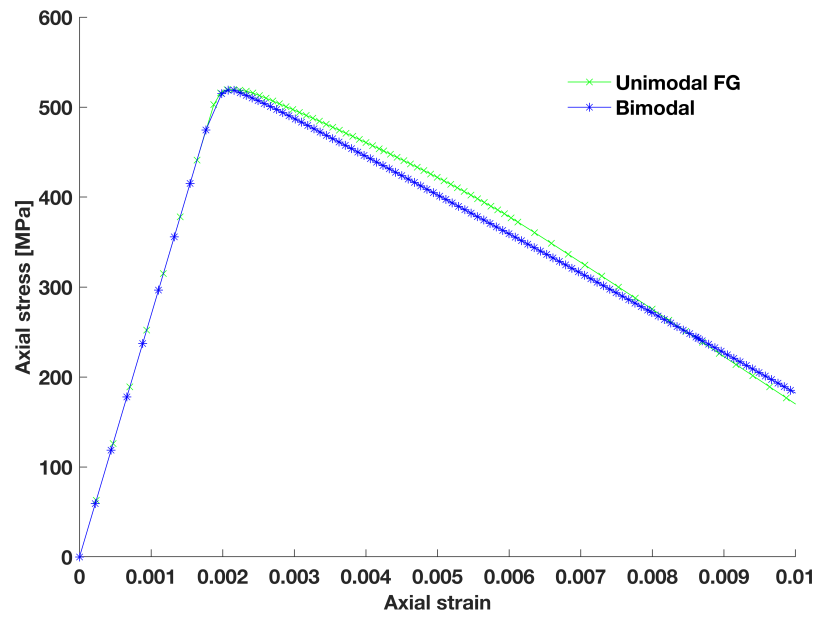

FIGURE 7: In the assumption of linear grains with damaging intergranular interfaces, the bimodal morphology has a light effect on the softening branch of the macro stress-strain curves.

and it would remain such both in the uni-modal or bi-modal case, as almost all orientations are represented in both cases.

Yet, as a plane perpendicular to the tensile direction is the most favourable location for crack initiation, microcracking naturally occurs first at the CG-FG interface in the bimodal case, on the top or the bottom of the CG. This also corresponds to the place where crystal plasticity predicts the smallest states of stress within the FG matrix. As experiments on 316L stainless steel have shown the positive effect of the presence of CGs on the elongation to fracture, these patterns of stress and strain interactions between FG and CG as provided by crystal plasticity may be considered as key factors in the micro-cracking process. Fracture analyses of the considered 316L steel should thus include not only the morphological characteristics of the grain boundaries but also the heterogeneous nature of stresses and strains resulting from the polycrystalline plasticity. This will correspond to future developments. 


\section{ACKNOWLEDGMENTS}

Ivano Benedetti acknowledges the support of INSA Rouen Normandie, where he has been invited as Foreign Visiting Professor between November and December 2019.

\section{REFERENCES}

1. B. Flipon, C. Keller, L. Garcia de la Cruz, E. Hug, and F. Barbe, "Tensile properties of spark plasma sintered AISI 316L stainless steel with unimodal and bimodal grain size distributions," Mater Sci Engng A 729, 248-256 (2018).

2. I. Benedetti and V. Gulizzi, "A grain-scale model for high-cycle fatigue degradation in polycrystalline materials," Int J Frac 116, 90-105 (2018).

3. E. Hall, "The deformation and ageing of mild steel: III discussion of results," Proc Phys Soc Section B 64, 747-753 (1951).

4. N. Petch, "The Cleavage Strength of Polycrystals," J Iron Steel Inst 174, 25-28 (1953).

5. M. Meyers, A. Mishra, and D. Benson, "Mechanical properties of nanocrystalline materials," Prog Mater Sci 51, 427-556 (2006).

6. A. Pineau, A. Benzerga, and T. Pardoen, "Failure of metals III: Fracture and fatigue of nanostructured metallic materials," Acta Mater 107, 508-544 (2016).

7. S. Ramtani, H. Bui, and G. Dirras, "A revisited generalized self-consistent polycrystal model following an incremental small strain formulation and including grain-size distribution effect," Int J Engng Sci 47, 537-553 (2009).

8. S. Berbenni, V. Favier, and M. Berveiller, "Impact of the grain size distribution on the yield stress of heterogeneous materials," Int J Plasticity 23, 114-142 (2007).

9. L. Zhu, S. Shi, K. Lu, and J. Lu, "A statistical model for predicting the mechanical properties of nanostructured metals with bimodal grain size distribution,” Acta Mater 60, 5762-5772 (2012).

10. B. Srinivasarao, K. Oh-ishi, T. Ohkubo, T. Mukai, and K. Hono, "Synthesis of high-strength bimodally grained iron by mechanical alloying and spark plasma sintering," Scripta Mater 58, 759-762 (2008).

11. G. Dirras, J. Gubicza, S. Ramtani, Q. Bui, and T. Szilágyi, "Microstructure and mechanical characteristics of bulk polycrystalline Ni consolidated from blends of powders with different particle size," Mater Sci Engng A 527, 1206-1214 (2010).

12. Z. Zhang, S. Vajpai, D. Orlov, and K. Ameyama, "Improvement of mechanical properties in SUS304L steel through the control of bimodal microstructure characteristics," Mater Sci Engng A 598, 106-113 (2014).

13. D. Tingaud, P. Jenei, A. Krawczynska, F. Mompiou, J. Gubicza, and G. Dirras, "Investigation of deformation micro-mechanisms in nickel consolidated from a bimodal powder by spark plasma sintering," Materials Characterization 99, 118-127 (2015).

14. B. Flipon, L. Garcia de la Cruz, E. Hug, C. Keller, and F. Barbe, "Elaboration of austenitic stainless steel with bimodal grain size distribution and investigation of their mechanical behavior," (20th Int. ESAFORM Conf. on Material Forming, 26-28 April 2017, Dublin, Ireland, AIP Conference Proceedings 1896, 200007, 2017).

15. J. Pipard, N. Nicaise, S. Berbenni, O. Bouaziz, and M. Berveiller, "A new mean field micromechanical approach to capture grain size effects," Comput Mater Sci 45, 604-610 (2009).

16. S. Ramtani, G. Dirras, and H. Bui, "A bimodal bulk ultra-fine-grained nickel: Experimental and micromechanical investigations," Mech Materials 42, 522-536 (2010).

17. X. Wang, F. Cazes, J. Li, A. Hocini, K. Ameyama, and G. Dirras, "A 3d crystal plasticity model of monotonic and cyclic simple shear deformation for commercial-purity polycrystalline ti with a harmonic structure," Mechanics of Materials 128, 117 - 128 (2019)

18. A. Magee and L. Ladani, "Representation of a microstructure with bimodal grain size distribution through crystal plasticity and cohesive interface modeling," Mech Mater 82, 1-12 (2015).

19. F. Lavergne, R. Brenner, and K. Sab, "Effects of grain size distribution and stress heterogeneity on yield stress of polycrystals: A numerical approach," Comput Mater Sci 77, 387-398 (2013).

20. B. Flipon, C. Keller, R. Quey, and F. Barbe, "A full-field crystal-plasticity analysis of bimodal polycrystals,” Int J Solids Struct 184, 178-192 (2020), doi:10.1016/j.ijsolstr.2019.02.005.

21. G. Sfantos and M. Aliabadi, "Multi-scale boundary element modelling of material degradation and fracture," Computer Methods in Applied Mechanics and Engineering 196, $1310-1329$ (2007)

22. I. Benedetti and M. Aliabadi, "A three-dimensional cohesive-frictional grain-boundary micromechanical model for intergranular degradation and failure in polycrystalline materials," Computer Methods in Applied Mechanics and Engineering 265, 36 - 62 (2013)

23. B. Flipon, L. Milhem, C. Keller, R. Quey, and F. Barbe, "Modelling of polycrystals using well-controlled voronoi-type tessellations and its applications to micromechanical analyses," (Presse des Mines, 2018) Chap. 23, pp. 187-198, physics and Mechanics of Random Media: from Morphology to Material Properties.

24. Neper, Neper: polycrystal generation and meshing, http://www.neper.info (2020).

25. R. Quey, P. Dawson, and F. Barbe, "Large-scale 3-D random polycrystals for the finite element method: Generation, meshing and remeshing," Comput Meth Appl Mech Engng 200, 1729-1745 (2011).

26. L. Méric and G. Cailletaud, "Single Crystal Modeling for Structural Calculations. Part 2: Finite Element Implementation," J Engng Mater Technol 113, 171-182 (1991).

27. M. H. Aliabadi, The boundary element method, volume 2: applications in solids and structures, Vol. 2 (John Wiley \& Sons, 2002).

28. V. Gulizzi, A. Milazzo, and I. Benedetti, "An enhanced grain-boundary framework for computational homogenization and micro-cracking simulations of polycrystalline materials," Computational Mechanics 56, 631-651 (2015).

29. V. Gulizzi and I. Benedetti, "Micro-cracking of brittle polycrystalline materials with initial damage," European Journal of Computational Mechanics 25, 38-53 (2016)

30. V. Gulizzi, C. Rycroft, and I. Benedetti, "Modelling intergranular and transgranular micro-cracking in polycrystalline materials," Computer Methods in Applied Mechanics and Engineering 329, 168 - 194 (2018) 
31. I. Benedetti, V. Gulizzi, and V. Mallardo, “A grain boundary formulation for crystal plasticity,” International Journal of Plasticity 83, 202 $224(2016)$

32. I. Benedetti, V. Gulizzi, and A. Milazzo, "Grain-boundary modelling of hydrogen assisted intergranular stress corrosion cracking," Mechanics of Materials 117, $137-151(2018)$

33. I. Benedetti and V. Gulizzi, "A grain-scale model for high-cycle fatigue degradation in polycrystalline materials," International Journal of Fatigue 116, $90-105$ (2018)

34. I. Benedetti, V. Gulizzi, and A. Milazzo, "A microstructural model for homogenisation and cracking of piezoelectric polycrystals," Computer Methods in Applied Mechanics and Engineering 357, 112595 (2019)

35. I. Benedetti and M. Aliabadi, "Multiscale modeling of polycrystalline materials: A boundary element approach to material degradation and fracture," Computer Methods in Applied Mechanics and Engineering 289, 429 - 453 (2015) 